\title{
The RNA-binding protein HuR is a novel target of Pirh2 E3 ubiquitin ligase
}

\author{
Alexandra Daks (1)', Alexey Petukhov,2, Olga Fedorova', Oleg Shuvalov', Alena Kizenko (1)', Elizaveta Tananykina', \\ Elena Vasileva (1) ${ }^{1}$, Oleg Semenov ${ }^{1}$, Andrew Bottrill $\mathbb{1}^{3}$ and Nickolai Barlev ${ }^{1,4}$
}

\begin{abstract}
The RING-finger protein Pirh2 is a p53 family-specific E3 ubiquitin ligase. Pirh2 also ubiquitinates several other important cellular factors and is involved in carcinogenesis. However, its functional role in other cellular processes is poorly understood. To address this question, we performed a proteomic search for novel interacting partners of Pirh2. Using the GST-pulldown approach combined with LC-MS/MS, we revealed 225 proteins that interacted with Pirh2. We found that, according to the GO description, a large group of Pirh2-associated proteins belonged to the RNA metabolism group. Importantly, one of the identified proteins from that group was an RNA-binding protein ELAVL1 (HuR), which is involved in the regulation of splicing and protein stability of several oncogenic proteins. We demonstrated that Pirh2 ubiquitinated the HuR protein facilitating its proteasome-mediated degradation in cells. Importantly, the Pirh2-mediated degradation of HuR occurred in response to heat shock, thereby affecting the survival rate of HeLa cells under elevated temperature. Functionally, Pirh2-mediated degradation of HuR augmented the level of c-Myc expression, whose RNA level is otherwise attenuated by HuR. Taken together, our data indicate that HuR is a new target of Pirh2 and this functional interaction contributes to the heat-shock response of cancer cells affecting their survival.
\end{abstract}

\section{Introduction}

Pirh2 (p53-induced RING-H2 protein) was first described as an androgen receptor $\mathrm{N}$-terminal interacting protein in $2002^{1}$. Human Pirh2 is coded by the $R C H Y 1$ gene (RING-finger and $\mathrm{CHY}$-zinc-finger domain-containing protein 1) and belongs to the RING-finger domain-containing proteins, many of which are E3 ligases. Accordingly, Pirh2 contains the centrally located RING domain responsible for the ubiquitin ligase activity, flanked by the $\mathrm{N}$ - and C-terminal domains responsible for proteinprotein interactions ${ }^{2}$.

Correspondence: Alexandra Daks (alexandra.daks@gmail.com) or

Nickolai Barlev (nick.a.barlev@gmail.com)

${ }^{1}$ Institute of Cytology, Russian Academy of Sciences, 194064 St Petersburg,

Russian Federation

${ }^{2}$ Almazov National Medical Research Centre, Institute of Hematology, 197341

St Petersburg, Russian Federation

Full list of author information is available at the end of the article

Edited by M. Piacentini
Pirh2 is a transcriptional target of the p53 tumor suppressor protein in mice. Similar to another p53-specific E3 ligase, Mdm2, Pirh2 is able to ubiqutinate p53 and target it for the proteasomal degradation ${ }^{3}$. Given the importance of the p53 for carcinogenesis, it is perhaps not surprising that Pirh2 is being studied mostly in the context of p53 suppression.

Besides p53, Pirh2 was shown to ubiquitinate two other members of the $\mathrm{p} 53$ protein family-p63 and $\mathrm{p} 73^{4-6}$. Pirh2, unlike MDM2, is able to degrade active p53 under the conditions of DNA damage, when p53 undergoes lysine-specific acetylation and methylation ${ }^{8}$. It is important to note that Pirh2 also mediates ubiquitination of mutant forms of the p53 protein, which are oncogenic, and the N-terminally truncated oncogenic isoform of p63 $(\Delta \mathrm{Np} 63)^{9,10}$. These data raise a question whether the cellular function of Pirh2 is oncogenic or tumor suppressive. To date, not much is known about the regulatory mechanisms of the RCHY1 gene expression. There are

\section{(c) The Author(s) 2021}

(c) (i) Open Access This article is licensed under a Creative Commons Attribution 4.0 International License, which permits use, sharing, adaptation, distribution and reproduction cc) in any medium or format, as long as you give appropriate credit to the original author(s) and the source, provide a link to the Creative Commons license, and indicate if changes were made. The images or other third party material in this article are included in the article's Creative Commons license, unless indicated otherwise in a credit line to the material. If material is not included in the article's Creative Commons license and your intended use is not permitted by statutory regulation or exceeds the permitted use, you will need to obtain permission directly from the copyright holder. To view a copy of this license, visit http://creativecommons.org/licenses/by/4.0/. 
only two transcription factors, p53 and its homolog p63, which were shown to control the RCHY1 gene expression $^{3,11}$.

Besides the p53 family members, Pirh2 is known to degrade a number of proteins playing key roles in such cellular processes as DNA damage response, cell cycle progression, gene expression regulation, and tumor transformation. These proteins include Poln, Chk2, p27 ${ }^{\mathrm{Kip} 1}$, and $\mathrm{HDAC} 1^{12-15}$.

Given the spectrum of Pirh2 targets, it is not surprising that Pirh2 is often implicated either as a biomarker and/or a prognostic factor in different types of cancer. In general, an elevated Pirh2 level is associated with tumor transformation, and a poor outcome and increased cancer aggressiveness. Accordingly, Pirh2 was shown to be upregulated in lung cancer ${ }^{16}$, prostate cancer ${ }^{12}$, and head and neck cancers ${ }^{17}$, and is associated with a poor prognosis of patients with glioma ${ }^{18}$, hepatocellular carcinoma $^{19}$, and oral squamous cell carcinoma ${ }^{20}$. In contrast, the analysis of genome-wide microarray data revealed that lower levels of Pirh2 mRNA are associated with a reduced survival of patients with breast and ovarian cancer, and lung squamous carcinomas ${ }^{21}$. Thus, the role of Pirh2 in carcinogenesis is still debatable and requires further elucidation.

There are 86 Pirh2-interacting proteins known to date, including those identified only by high-throughput screenings without further validation. In drastic contrast, there are 522 known interacting partners for the p53 ubiquitin ligase, MDM2 (https://thebiogrid.org/), which clearly indicates that the interactome of Pirh2 is underestimated. To broaden our knowledge about the p53independent role of Pirh2 in tumor transformation and other physiological processes, we performed a proteomic search for additional Pirh2-interacting partners. Using various experimental approaches, we demonstrated that Pirh2 was involved in the regulation of the RNA-binding protein, HuR1 (ELAVL1), thereby affecting the mRNA stability of its target genes, including $c-M y c$.

\section{Materials and methods Plasmids}

The pcDNA-Pirh2 construct was obtained from Dr S. Benchimol. For expressing Pirh2-3×FLAG and HuR$3 \times$ FLAG, the pIRES-hrGFP-1a vector (Agilent Technologies, Santa Clara, CA, USA) was used. For expressing Pirh2-GST and HuR-GST in Escherichia coli, the corresponding inserts were cloned into the pGEX-5X-1 vector backbone (GE Healthcare, Milwaukee, WI, USA) and were subsequently purified on Glutathione $4 \mathrm{~B}$ sepharose (GE Healthcare, Milwaukee, WI, USA). The Ku70-GST protein was obtained as described previously ${ }^{22}$. For ubiquitination assay experiments, the pcDNA-6His-Ubiquitin vector was used. An empty pcDNA3.1 vector was used as negative control. For stable Pirh2 knockdown (KD), the pLKO.1-TRC vector ${ }^{23}$ with a Pirh2-specific short hairpin RNA (shRNA) insert ${ }^{24}$, the lentiviral packaging plasmid psPAX2 (Addgene \#12260), and the envelope plasmid pMD2.G (Addgene \#12259) were used. For HuR KD, the following shRNA were used: ELAVL1_top 5'-CCGGCG AGCTCAGAGGTGATCAAAGCTCGAGCTTTGATCA CCTCTGAGCTCGTTTTTG-3' and ELAVL1_bot 5'AATTCAAAAACGAGCTCAGAGGTGATCAAAGCTC GAGCTTTGATCACCTCTGAGCTCG-3'. For stable Pirh2 overexpression (Pirh2 OE), LeGO-iG2 vector ${ }^{25}$ was used.

\section{Cell cultures}

All cell lines were cultured in standard conditions, using appropriate culture media supplemented with $10 \%$ fetal bovine serum (Walkersville, MD, USA), 100 units $/ \mathrm{ml}$ penicillin, $100 \mathrm{mg} / \mathrm{ml}$ streptomycin, and $2 \mathrm{mM}$ L-glutamine. Human embryonic kidney (HEK293T), human breast adenocarcinoma (MDA-MB-231), and human cervix adenocarcinoma (HeLa) cells were cultured in Dulbecco's modified Eagle's medium. For human nonsmall cell lung carcinoma (H1299) cells, RPMI 1640 medium was used. Cells were grown at $37^{\circ} \mathrm{C}$ in $5 \% \mathrm{CO}_{2}$ humidified atmosphere. $\mathrm{H} 1299$ cells were transfected using X-tremeGENE HP reagent (Sigma Aldrich, St. Louis, MO, USA) and HEK293T cells were transfected using TurboFect (Thermo Fisher Scientific, Waltham, MA, USA) according to the manufacturer's instructions. H1299, HeLa, and MDA-MB-231 cell lines stably overexpressing Pirh2, and H1299 and HeLa cell lines with Pirh2 KD were obtained using the protocol described previously $^{24}$. All cell lines were obtained from ATCC and were checked regularly for mycoplasma infections.

\section{GST-pulldown assay and mass spectrometry}

Glutathione-S-transferase (GST)-pulldown assay was performed using the previously developed approach ${ }^{26,27}$. Briefly, the Pirh2-GST recombinant protein and an appropriate amount of GST were immobilized on glutation-sepharose beads (GE Healthcare, Milwaukee, WI, USA), which were then added to the cell lysate and incubated with rotation at $4{ }^{\circ} \mathrm{C}$ for $3 \mathrm{~h}$. After washing with phosphate-buffered saline (PBS), retained proteins were eluted and subjected to either mass spectrometry (MS) analysis or western blotting for detection. The MS analysis was carried out as described previously ${ }^{27}$. Briefly, the proteins bound to GST or Pirh2-GST respectively were separated by SDS-polyacrylamide gel electrophoresis, excised from the gel, and subjected to trypsin digest in the gel. Liquid chromatography-tandem MS (LC-MS/MS) was carried out using an RSLCnano high-performance liquid chromatography system (Dionex, Sunnyvale, CA, 
USA) and an LTQ-Orbitrap-Velos mass spectrometer (Thermo Fisher Scientific, Waltham, MA, USA).

\section{Co-immunoprecipitation}

The co-immunoprecipitation protocol was described previously ${ }^{28}$. Briefly, HEK293T cells were transiently transfected with an empty pIRES-hrGFP-1a vector and the vector coding Pirh2-3×FLAG. Forty-eight hours after transfection, the cells were collected for lysates preparation with a modified radioimmunoprecipitation assay buffer. The lysates were then incubated with anti-FLAG M2 agarose beads (Sigma Aldrich, St. Louis, MO, USA) for $4 \mathrm{~h}$ at $4{ }^{\circ} \mathrm{C}$ with rotation. Following washes, the bound proteins were eluted by a $3 \times$ FLAG peptide solution and analyzed by western blotting.

Co-immunoprecipitation of endogenous Pirh 2 and $\mathrm{HuR}$ proteins was carried out in extract made from $1 \times 10^{7}$ HeLa cells essentially as described in ref. ${ }^{29}$. Two equal portions of cell lysate were incubated with $5 \mu \mathrm{g}$ anti-HuR mouse monoclonal antibodies and equal amount mouse IgG as a negative control at $4{ }^{\circ} \mathrm{C}$ for $4 \mathrm{~h}$ with rotation. Protein $\mathrm{G}$ agarose magnetic beads (Thermo Fisher Scientific, Waltham, MA, USA) was pre-incubated with $0.1 \%$ bovine serum albumin for $1 \mathrm{~h}$ to minimize the unspecific binding. Then, pre-incubated Protein G agarose was added to cell lysates containing antibodies and incubated at $4{ }^{\circ} \mathrm{C}$ for $1 \mathrm{~h}$ with rotation. After the incubation, the beads were washed three times with ice-cold lysis buffer using a magnet. The bound proteins were analyzed by western blotting using temperature-denaturated co-IP samples in Laemmli buffer.

\section{Ubiquitination assay}

In cellulo ubiquitination was performed as previously described $^{30}$. HEK293T cells were transiently transfected with pIRES-HuR, pcDNA-6His-Ubiquitin, and pcDNAPirh2 vectors. An empty pcDNA3.1 plasmid was used as negative control. Twenty-four hours after transfection, cells were treated with $10 \mu \mathrm{M}$ MG132 for $16 \mathrm{~h}$ and then lysed in $8 \mathrm{M}$ urea-containing buffer $(\mathrm{pH}$ 8.0). 6Hisubiquitinated proteins were purified on Ni-NTA beads (Qiagen, Hilden, Germany). The bound proteins were eluted and analyzed by western blotting.

In vitro ubiquitination assay was performed using Ubiquitinylation kit (BML-UW9920; Enzo Biochem, New York, NY, USA) according to the manufacturer's recommendations. The purified GST-tagged proteins were used for in vitro ubiquitination reaction: HuRGST was used as a substrate; full-length Pirh2 (Pirh2 FL-GST), N-terminal domain of Pirh2 (Pirh2 NTDGST), and GST as the negative control were used for their E3 in vitro ligase activity investigation. The protein ratios used for the reaction are demonstrated in Supplementary Fig. S5.

\section{RNA immunoprecipitation}

To detect RNAs associated with the HuR protein, RNA immunoprecipitation (RIP) was performed. H1299 cells $\left(8 \times 10^{6}\right)$ were collected by trypsinization and resuspended in ice-cold RIP buffer containing $150 \mathrm{mM} \mathrm{KCl}, 25 \mathrm{mM}$ Tris- $\mathrm{HCl} \mathrm{pH} 7.4,5 \mathrm{mM}$ EDTA, $0.5 \mathrm{mM}$ dithiothreitol, $0.5 \% \mathrm{NP}-40, \quad 100 \mu \mathrm{g} / \mathrm{ml}$ RNAse inhibitor RiboLock (Thermo Fisher Scientific, Waltham, MA, USA), and protease inhibitor cocktail (Sigma Aldrich). After 10 min incubation in the RIP buffer and centrifugation at $13,000 \times g$ for $5 \mathrm{~min}$, supernatant was collected and used for subsequent manipulations. Seven micrograms of antiHUR antibodies (3A2, Santa Cruz Biotechnology, Santa Cruz, CA, USA) were added to the supernatant and incubated for $4 \mathrm{~h}$ at $4{ }^{\circ} \mathrm{C}$ followed by incubation with $40 \mu \mathrm{l}$ of protein $\mathrm{G}$ magnetic beads (Thermo Fisher Scientific, Waltham, MA, USA) per sample for $1 \mathrm{~h}$ at $4{ }^{\circ} \mathrm{C}$ with rotation. After the incubation, the beads were washed three times with ice-cold RIP buffer using a magnet and then RNA was isolated by Trizol reagent (Thermo Fisher Scientific, Waltham, MA, USA) according to the manufacturer's instruction. Total RNA calculated according to the number of cells was used as input control. To analyze the immunoprecipitated mRNA, reverse-transcriptase PCR (RT-PCR) was performed. cDNA was synthetized using Reverse Transcription Kit (Thermo Fisher Scientific, USA) according to the manufacturer's recommendations. Quantitative PCR (qPCR) was performed using primers for coding sequence (CDS) and $3^{\prime}$-untranslated region ( $3^{\prime}$-UTR) of the c-Myc mRNA sequence. The following primers were used: $\mathrm{c}-\mathrm{Myc}$ CDS Fwd-5'-CTCCT CCTCGTCGCAGTAGA-3', c-Myc CDS Rev-5'-GCTGC TTAGACGCTGGATTT-3'; c-Myc 3'-UTR Fwd-5'-AAC CTTGGCTGAGTCTTGAG-3', Rev-5'-AGTTCTTTTAT GCCCAAAGTCCA-3'. Glyceraldehyde 3-phosphate dehydrogenase (GAPDH) signal was used as a reference and normalization control.

\section{Colony-formation assay}

The assay was performed essentially as described previously ${ }^{24}$. Five hundred cells, both treated at $45^{\circ} \mathrm{C}$ and untreated, were seeded in RPMI 1640 medium on $30 \mathrm{~mm}$ plates and incubated for 7 days for colony formation. The colonies were fixed and stained for $10 \mathrm{~min}$ with fixing/ staining solution containing $0.05 \%$ crystal violet, $1 \%$ formaldehyde, and $1 \%$ methanol buffered with PBS. Following washes and drying, the colonies were scored and analyzed manually. The experiments were performed in triplicates.

\section{Cell cycle analysis}

Cell cycle analysis was performed as previously described $^{31}$. Briefly, collected cells were washed twice with PBS followed by incubation with $1 \%$ saponin for $20 \mathrm{~min}$. Then, 
DNA was treated by $1 \mathrm{mg} / \mathrm{ml}$ RNase A and stained with $50 \mathrm{mg} / \mathrm{ml}$ propidium iodide for $30 \mathrm{~min}$. Flow cytometry was performed using the CytoFLEX instrument (Beckman Coulter, Inc., Miami, FL, USA). Analysis was carried out using CytExpert Software.

\section{Real-time cell proliferation assay}

These tests were performed using the xCELLigence system (ACEA Biosciences, San Diego, CA, USA) according to the manufacturer's instruction. For cell proliferation assays, $2 \times 10^{4}$ cells were seeded in each well of E-plate 16 (ACEA Biosciences, San Diego, CA, USA) in RPMI 1640 medium. Cell index was registered every $15 \mathrm{~min}$.

\section{Real-time PCR}

To assess the mRNA levels, total RNA was extracted from cells using Trizol reagent (Sigma Aldrich, St. Louis, MO, USA). cDNA was synthetized using Reverse Transcription Kit (Thermo Fisher Scientific, Waltham, MA, USA) according to the manufacturer's recommendations. qPCR was performed after cDNA synthesis using previously published primers ${ }^{24,28}$. For $\mathrm{HuR}$ analysis, the following primers were used: forward $5^{\prime}$-GAGGCTCCAGT CAAAAACCA- $3^{\prime}$ and reverse $5^{\prime}$-GTTGGCGTCTTTG ATCACCT- $3^{\prime}$. mRNA expression levels were calculated relative to GAPDH by $\Delta \Delta \mathrm{Ct}$ method.

\section{Western blotting}

For western blot analysis (WB), whole-cell extracts were prepared unless otherwise specified. The primary antibodies against the analyzed proteins were used as follows: Pirh2 (EPR14980, Abcam), $\beta$-actin (A3854, Sigma Aldrich, St. Louis, MO, USA), c-Myc (9402S, Cell Signaling Technology, Beverly, MA, USA), FLAG (F1804, Sigma Aldrich, St. Louis, MO, USA), HuR (12582S, Cell Signaling Technology, Beverly, MA, USA), $\beta$-tubulin (T8535, Sigma Aldrich, St. Louis, MO, USA), and His-Tag (2365, Cell Signaling Technology, Beverly, MA, USA); secondary antibodies used were anti-mouse and antirabbit (Sigma Aldrich, St. Louis, MO, USA).

\section{Bioinformatics}

Correlations of expression levels of RCHY1 (Pirh2) and $m y c$ (c-Myc) with the survival rates of lung cancer patients were calculated by algorithms described by Amelio et al. ${ }^{32}$. The individual contribution of ELAVL1 $(\mathrm{HuR})$ was examined as described in Antonov et al. ${ }^{33}$. using the Gene Expression Omnibus (GEO) microarray data.

\section{Statistical analysis}

Data are shown as mean \pm SD or SEM of at least three replicates. Statistical significance was analyzed using
Student's $t$-test. $P<0.05$ was considered significant. $P<$ 0.01 is denoted as an asterisk ":".

\section{Results \\ Identification of novel Pirh2-interacting proteins}

To identify the proteins that interact with Pirh2 and, hence, may represent its potential targets for ubiquitination, we used the GST-pulldown approach combined with high-resolution MS (Fig. 1). To this end, the recombinant protein Pirh2-GST was incubated with the cell extract prepared from the human embryonic kidney cell line HEK293T (Fig. 1A). Native GST protein was used as control for nonspecific binding. The Pirh2-GST-bound proteins were identified by LC-MS/MS.

The MS analysis has yielded 346 interacting proteins in total: 50 proteins interacted with GST only, 256 proteins interacted specifically with Pirh2-GST, and 40 proteins interacted with both GST and Pirh2-GST. The raw data were published in the Mendeley Datasets Repository (https://doi.org/10.17632/24bh4cvhzd.1). The data processing consisted of excluding prokaryotic proteins, keratins, Pirh2 protein, and GST from the list of interacting proteins. Each identified protein, for which at least three peptides were detected (score $\geq 3$ ), and which either did not bind or bound poorly GST compared to Pirh2-GST, was defined as significant. After processing the data obtained, we revealed 225 proteins bound to Pirh2, but not GST (Fig. 1B and Supplementary Table S1).

We grouped the identified proteins according to their functions in the cell. As a result, we found that almost half of the identified proteins belong to "Ribosomes and translation" and "RNA processing and splicing" groups (Fig. 1C, D). In total, we combined all identified proteins into 15 groups, with several representative proteins from each group being shown in Fig. 1C, D. Among the Pirh2interacting proteins were the ones that are involved in gene expression regulation, DNA repair, apoptosis, and tumor transformation (e.g., PARP1, ku70, Sirt1, HuR, and Dicer).

To validate our GST-pulldown results, we chose one of the highest $(\mathrm{HuR})$ and one of the lowest scoring proteins (Ku70), and carried out reciprocal pulldowns of Pirh2 using the Ku70-GST or HuR-GST recombinant proteins. Both HuR-GST and Ku70-GST, but not GST alone, were able to bind Pirh2 (Supplementary Fig. S1). Thus, we confirmed the specificity of protein-protein interactions with Pirh2 in our assay.

\section{Pirh2 interacts with and ubiquitinates the RNA-binding protein HuR leading to its proteasomal degradation}

As our results clearly suggested that Pirh2 is important for the expression of proteins, we questioned whether Pirh2 can regulate the mRNA stability, thereby indirectly affecting the protein biosynthesis. It is noteworthy that 

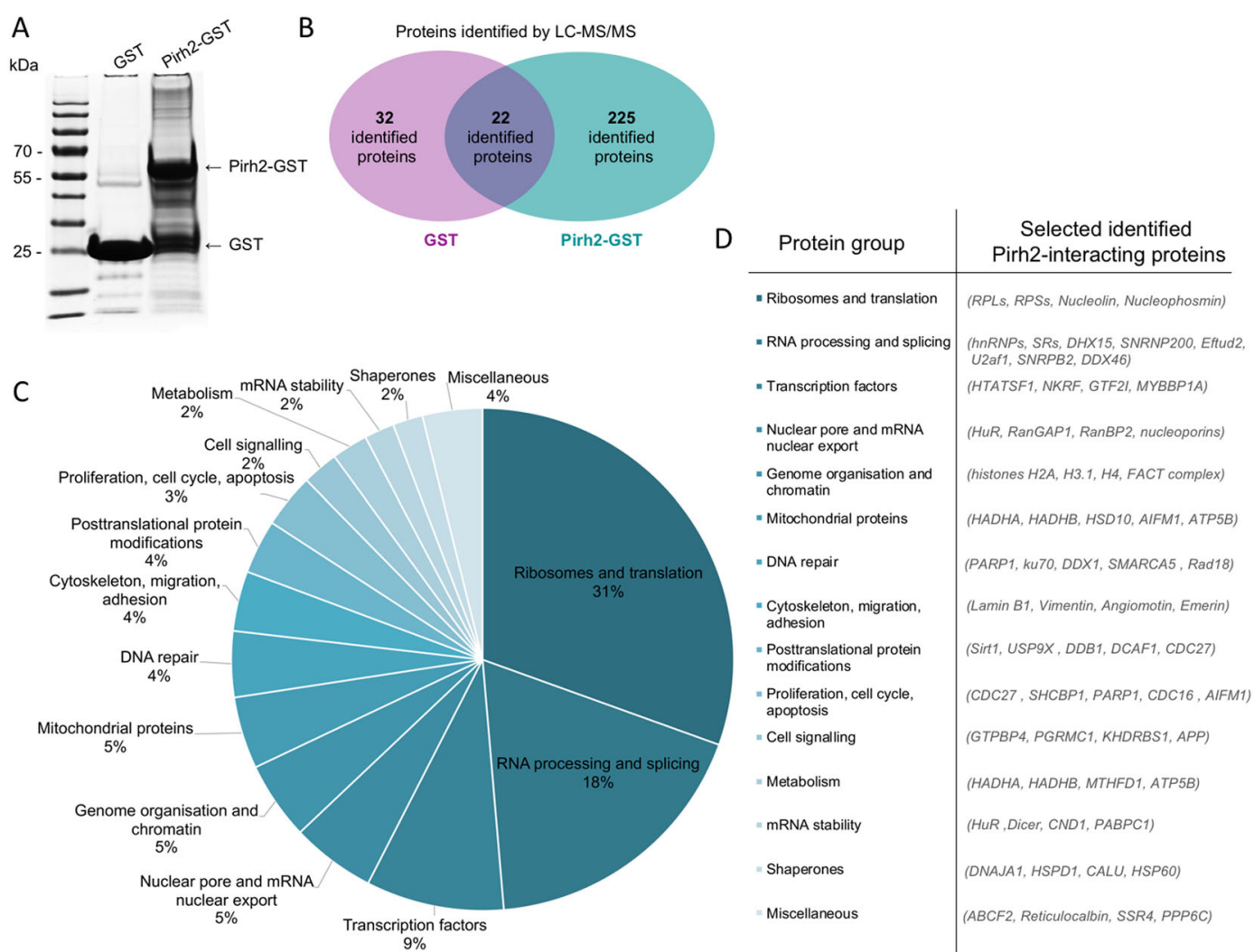

Fig. 1 Interactome of Pirh2. A Coomassie-stained gel of GST and Pirh2-GST proteins after incubation with cell extracts from HEK293T. Pirh2-GST fusion protein and native GST protein are denoted with arrows. The mass spectrometry data were processed using Mascot 49 and Scaffold 4 software. B Euler diagram demonstrating the number of GST- and Pirh2-GST-interacting proteins identified by LC-MS/MS. C A pie chart of identified Pirh2interacting proteins grouped by their functions. D The list of functionally distinguished groups with selected representative proteins that interact with Pirh2.

HuR, a global regulator of mRNA stability, has emerged as one of the strong Pirh2 interactors (Fig. 2). Thus, we decided to investigate the functional significance of this interaction. First, we performed reciprocal GST-pulldown assay using the purified HuR-GST protein and the cellular extract prepared from HEK293T cells. We demonstrated that HuR-GST binds the endogenous Pirh2 protein more strongly compared to GST, supporting the previously obtained LC-MS/MS data (Fig. 2A).

To determine the portion of Pirh2 responsible for HuR binding, we employed GST-pulldown assay. To this end, purified GST-tagged deletion mutants of Pirh2 (the Nterminal domain, 1-137 a.a. (NTD-GST), the RING domain, 138-189 a.a. (RING-GST), and the C-terminal domain, 190-261 a.a. (CTD-GST)) were incubated with the whole-cell extract from HEK293T. The full-length GST-Pirh2 protein and GST alone were also analyzed as positive and negative controls, respectively. Results of the binding experiment (Fig. 2B) clearly suggest that the Nterminal and RING domains of Pirh2 are responsible for the Pirh2-HuR interaction.
To confirm the GST-pulldown interaction results, we performed reciprocal co-immunoprecipitation of endogenously expressed HuR and ectopic Pirh $2-3 \times$ FLAG or, conversely, endogenously expressed Pirh 2 and ectopic HuR-3×FLAG. We found that that Pirh2 specifically interacted with HuR in cellulo (Fig. 2C, D).

We also confirmed the Pirh2-HuR interaction on the endogenous level by co-immunoprecipitation. The endogenous Pirh2-HuR complex from HeLa cells was precipitated using anti-HuR monoclonal antibodies (Fig. 2E). Taken together, these results confirm an existence of the Pirh2-HuR interaction in cells.

Next, we analyzed whether Pirh2 affects the HuR protein level in cells. To test this, we transfected H1299 cells with increasing amounts of the Pirh2-expressing plasmid. The total amount of DNA in each sample was normalized with an empty pcDNA3.1 plasmid. As shown in Fig. 3A, increasing levels of Pirh2 expression led to a concomitant reduction of the HuR level. We also tested the effect of Pirh2 on HuR in another tumor cell line, MDA-MB-231, which constitutively expressed either control plasmid 
A

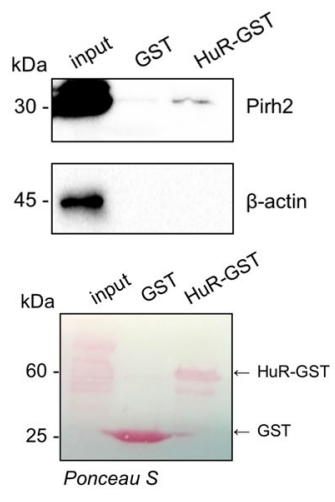

B

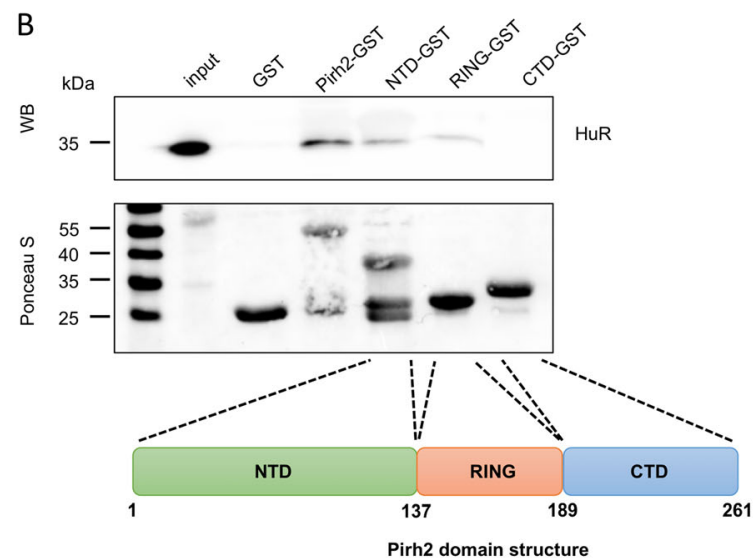

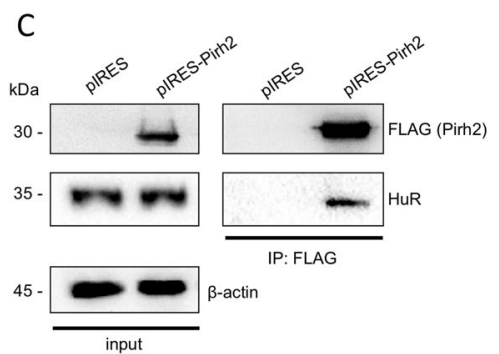
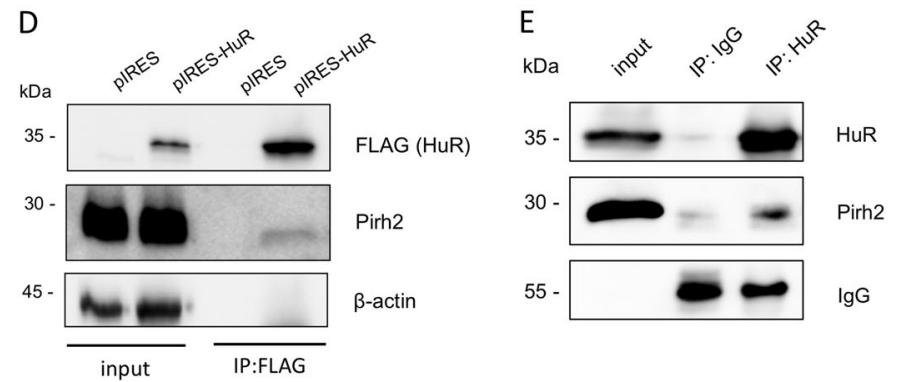

Fig. 2 Pirh2 binds to HuR in vitro and in cellulo. A HUR-GST interacts with Pirh2. GST-pulldown assay with extracts from HEK293T, demonstrating the interaction of HuR-GST with Pirh2. A Ponceau-stained membrane. HuR-GST fusion protein and native GST protein are denoted with arrows (left); western blot analysis of Pirh2 binding to GST and HuR-GST (right). B Identification of the Pirh2 domains involved in HuR binding. WB analysis of GSTpull-down assay with purified GST-tagged proteins: GST, full-length Pirh2 (Pirh2-GST), the N-terminal domain of Pirh2 1-132 a.a. (NTD-GST), the RING domain of Pirh2 138-189 a.a. (RING-GST), and the C-terminal domain of Pirh2 190-261 a.a. (CTD-GST) (upper panel); the scheme demonstrating the Pirh2 domain structure (bottom panel). C Pirh2 interacts with HuR in cellulo. Western blot analysis of co-immunoprecipitation in HEK293T cells of ectopically expressed Pirh2-3xFLAG (pIRES-Pirh2) and endogenous HuR. An empty pIRES vector was used as negative control. D HuR interacts with Pirh2 in cellulo. Western blot analysis of co-immunoprecipitation in HeLa cells of ectopically expressed HuR-3XFLAG (pIRES-HuR) and endogenous Pirh2. An empty pIRES vector was used as negative control. E Co-immunoprecipitation of the endogenous HuR and Pirh2 proteins using anti-HuR monoclonal antibodies.

(LEGO) or Pirh2 (LEGO-Pirh2). Expectedly, constitutive overexpression of Pirh2 decreased the protein level of HuR (Supplementary Fig. S2).

To investigate whether the effect of Pirh2 on HuR involved its protein stability, we performed the cycloheximide-mediated blockade of the protein synthesis. To this end, H1299 cells transfected with the Pirh2-coding plasmid or the empty vector were used. As shown on Fig. 3B, ectopic expression of Pirh2 attenuated the level of HuR expression and ultimately reduced the half-life of the protein from 16 to $8 \mathrm{~h}$. Thus, we confirmed that Pirh2 affected the protein level of HuR in different cell types. The fact that adding a proteasome inhibitor MG132 to HeLa cells stabilized the protein level of HuR even in the presence of Pirh2 indicated that $\mathrm{HuR}$ attenuation likely involved the ubiquitindependent proteasome system (Fig. 3C).

As Pirh2 is the E3 ubiquitin ligase that polyubiquitinates its targets leading to their proteasomal degradation, we assumed that HuR may be a novel substrate for Pirh2dependent ubiquitination. To test this hypothesis, we performed ubiquitination of $\mathrm{HuR}$ in HeLa cells expressing 6His-ubiquitin and different levels of Pirh2 (Fig. 3D). Ectopic overexpression of Pirh2 increased the level of polyubiquitinated $\mathrm{HuR}$, hence suggesting that $\mathrm{HuR}$ is a potential target of Pirh2-dependent ubiquitination. We also repeated the ubiquitination assay in HEK293T cells with essentially similar results, i.e., Pirh2 was able to ubiquitinated HuR (Supplementary Fig. S3). To address the question of Pirh2 involvement in ubiquitination of HuR, we also compared the efficacies of in cellulo ubiquitination of the endogenous HuR protein in the presence of the full-length or mutant Pirh2 (Pirh2 NTD) proteins (Supplementary Fig. S4A). Indeed, the full-length Pirh2 protein ubiquitinated HuR more efficiently compared to the Pirh2 NTD mutant protein. We also confirmed the inability of catalytically inactive mutant of 

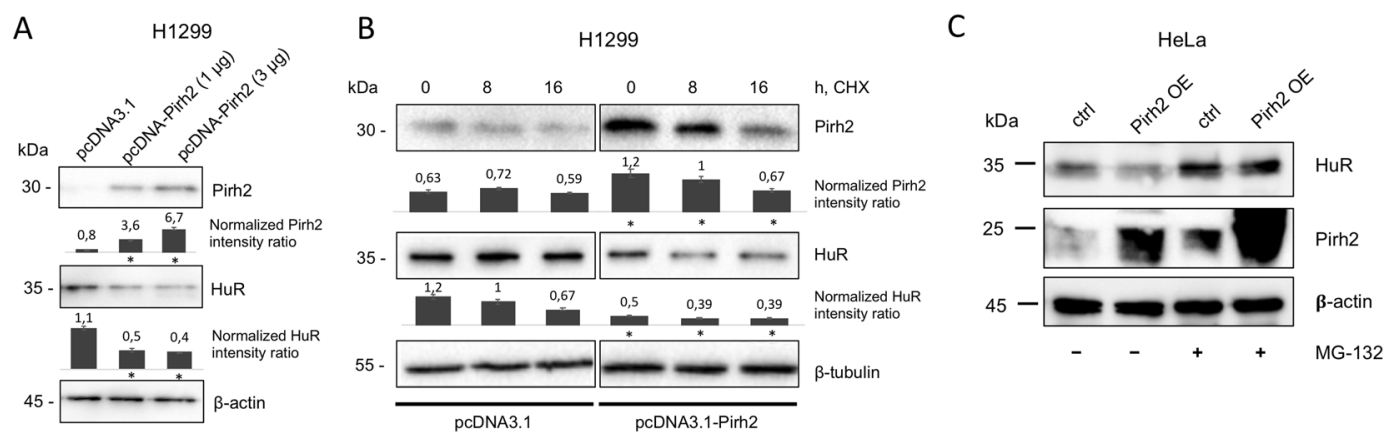

D

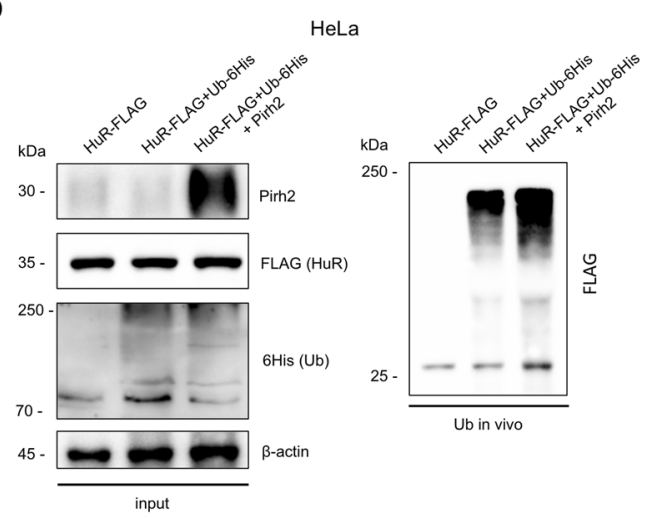

$\mathrm{E}$
Ubiquitinilation in vitro

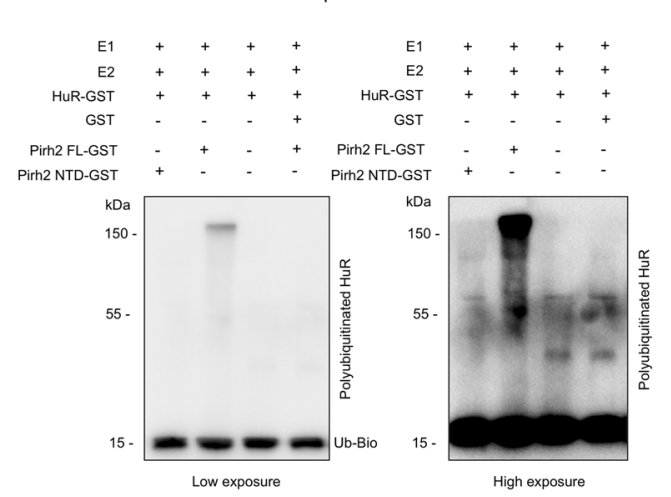

Fig. 3 Pirh2 downregulates HuR level via ubiquitination and targeting for the degradation. A Pirh2 decreases HuR level in H1299 cells. Transfection of H1299 cells with different quantity of pcDNA3-Pirh2 plasmid proportionally decreases HuR level. Empty pcDNA3.1 was used as a control and to keep the total plasmid DNA amount $3 \mu \mathrm{g}$ in each sample. The normalized intensity ratios were calculated as ratios between the signals of proteins analyzed and the corresponding actin bands on the basis of three measurements. Error bars indicate \pm SD. ${ }^{*} p \leq 0.05$ vs. empty vector transfection according to Student's t-test. B Pirh2 shortens half-life time of HuR. Western blot analysis of HuR protein level in H1299 cells transfected with pcDNA-Pirh2 and pcDNA3.1 empty vector, and treated with $50 \mu \mathrm{M}$ cycloheximide (CHX) for 8 and $16 \mathrm{~h}$. The normalized intensity ratios were calculated as ratios between the signals of proteins analyzed and the corresponding actin bands on the basis of three measurements. Error bars indicate \pm SD. ${ }^{*} p \leq 0.05$ vs. empty vector transfection for the corresponding time points according to Student's $t$-test. C WB analysis of HuR level in HeLa cells transiently transfected with pcDNA3-Pirh2 plasmid (Pirh2 OE) and pcDNA3.1 as a control (ctrl). The cells were treated with $10 \mu M$ MG132 for $16 \mathrm{~h}$. D Pirh2 ubiquitinates HuR in cellulo. Western blot analysis of Ni-agarose-precipitated 6 His-ubiquitinated HuR protein from HeLa cells transfected with pIRES-HuR (HuR-3XFLAG); pIRES-HuR and pcDNA-6His-Ubiquitin (Ub-6His); and pIRES-HuR, pcDNA-6His-Ubiquitin, and pcDNA-Pirh2 (Pirh2). pcDNA3.1 vector was used to keep the same amount of total plasmid DNA in each sample (right). Western blot analysis of Pirh2 and HuR3XFLAG in inputs (left). $\mathbf{E}$ In vitro ubiquitinilation assay. Ubiquitin-activating enzyme (E1), UbcH5b (E2) and GST-tagged HuR (HuR-GST, substrate), and biotin-tagged ubiquitin were added in equal amounts in each portion of the reaction. GST, full-length Pirh2 (Pirh2 FL-GST), and the catalytically inactive N-terminal domain of Pirh2 1-132 a.a. (NTD-GST) were tested for their ability to ubiquitinate HuR. No E3 ligase control was also used. The WB analysis of the ubiquitinilation reaction was performed using anti-biotin antibodies. Two variants of exposure are shown for better illustration. The relative amounts of purified proteins used in the reaction are demonstrated in Supplementary Fig. S5.

Pirh2 to downregulate the HuR protein level (Supplementary Fig. S4B).

Furthermore, to prove that Pirh2 is involved in direct ubiquitination of HuR, we performed in vitro ubiquitination assay using purified GST-tagged full-length Pirh2 (Pirh2 FL-GST) and the catalytically inactive Pirh2 mutant (Pirh2 NTD-GST). We also used a non-fused GST protein as a negative control. As shown in Fig. 3E, efficient ubiquitination of HuR occurs only in the presence of the full-length Pirh2 protein.

Thus, we demonstrated that Pirh2 was able to interact and ubiquitinate the RNA-binding protein HuR leading to its destabilization on the protein level.
Pirh2 affects HuR stability during the heat-shock response

It is well established that HuR protein plays a role in heat-shock response affecting the mRNA stability and cellular localization of its target genes, including mRNA of $\mathrm{HSP}^{34-36}$. In addition, HuR was shown to form cytoplasmic stress granules in response to heat shock (HS), yet its total level in the cell decreased ${ }^{37}$. Therefore, to assess the physiological significance of Pirh2-mediated attenuation of $\mathrm{HuR}$, we examined how Pirh2 affected the stability of HuR under the heat-shock stress conditions.

We asked a question of whether Pirh2 via attenuating the HuR stability may affect cell survival after the treatment with sublethal HS. We performed colony-formation 

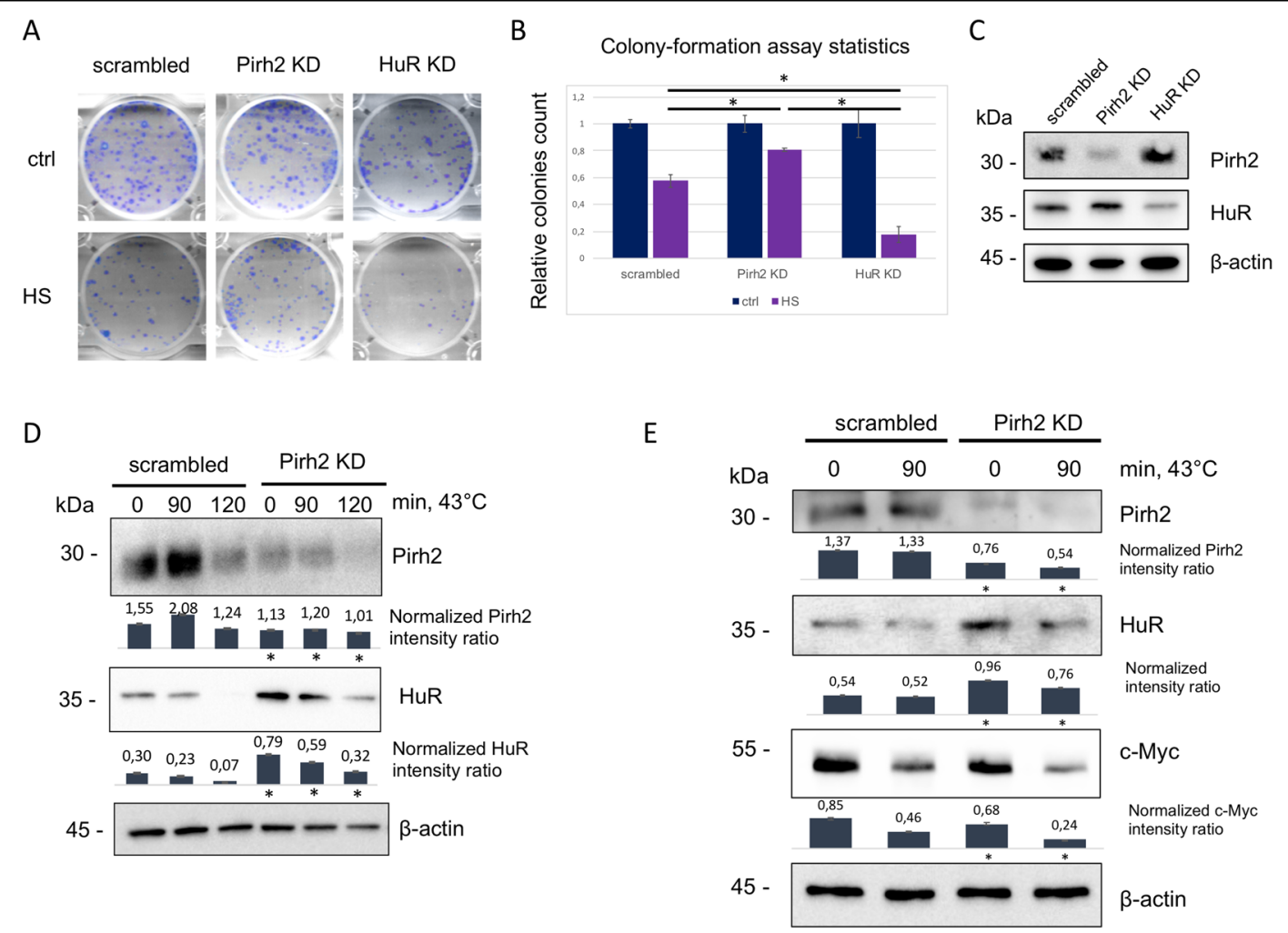

Fig. 4 Pirh2 affects HuR stability, cellular survival, and cell cycle in HeLa cells during heat-shock response. A Knockdown of Pirh2 leads to an increase of HeLa cell viability after heat-shock exposition. Colony-formation assay of Pirh2 knockdown (Pirh2 KD), HuR knockdown (HuR KD), and control (scrambled) HeLa cells in normal condition (ctrl) and after $45^{\circ} \mathrm{C}$ incubation for $3 \mathrm{~h}(\mathrm{HS})$. The test was performed in triplicates. B Statistical analysis of colony-formation assay. Error bars indicate \pm SEM. C Western blot analysis demonstrating protein levels of Pirh2 and HuR in Pirh2 knockdown (Pirh2 KD), HuR knockdown (HuR KD), and control (scrambled) HeLa cells. D Pirh2 destabilizes HuR under heat shock. Western blot analysis of HuR level in HeLa cells with Pirh2 knockdown (Pirh2 KD) and control HeLa cells (scrambled) after incubating at $43^{\circ} \mathrm{C}$ for 90 and 120 min. The normalized intensity ratios were calculated as ratios between the signals of proteins analyzed and the corresponding actin bands on the basis of three measurements. Error bars indicate $\pm \mathrm{SD}$. ${ }^{*} p \leq 0.05 \mathrm{vs}$. scrambled for the corresponding time points according to Student's $t$-test. $\mathbf{E}$ Pirh2 stabilizes c-Myc protein in HeLa cells. Western blot analysis of Pirh2, HuR, and c-Myc levels in HeLa cells with Pirh2 knockdown (Pirh2 KD) and control HeLa cells (scrambled), both in normal condition and after incubating at $43^{\circ} \mathrm{C}$ for $90 \mathrm{~min}$. The normalized intensity ratios were calculated as ratios between the signals of proteins analyzed and the corresponding actin bands on the basis of three measurements. Error bars indicate $\pm S D$. ${ }^{*} p \leq$ 0.05 vs. scrambled for the corresponding time points according to Student's $t$-test.

assay to test the viability of HeLa cells with suppressed Pirh2 or HuR expression compared to control cells after $45^{\circ} \mathrm{C}$ heat-shock exposure for $3 \mathrm{~h}$. Pirh2 KD led to a statistically significant increase in the number of colonyforming units compared to control cells, whereas KD of HuR had the opposite effect (Fig. 4A, B). The efficiency of Pirh2 and HuR KDs is demonstrated in Fig. 4C. We have also performed cell cycle analysis of HeLa cells with Pirh2 and HuR KD exposed to $\mathrm{HS}\left(45^{\circ} \mathrm{C}, 2 \mathrm{~h}\right)$ followed by $16 \mathrm{~h}$ of recovery. In response to the heat-shock treatment, the amount of Pirh2 KD cells in the G2 phase was higher compared to control cells, whereas HuR KD cells displayed the opposite effect (Supplementary Fig. S6). As the sublethal dose of HS causes proteotoxic stress that mainly results in the G2/M cell cycle arrest ${ }^{38,39}$, we concluded that KD of Pirh2, which leads to an increased G2 arrest, correlated with cell survival.
Next, we investigated whether Pirh2 attenuated the level of HuR upon HS in HeLa cells. As shown in Fig. 4D, protein levels of both HuR and Pirh2 decreased upon the exposure of cells to high temperature. However, in HeLa cells with KD Pirh2, the level of HuR was higher at all the time points compared to the matching control cells. To address the question of whether KD of Pirh2 augments the amount of cellular HuR by increasing its mRNA level, we performed qRT-PCR analysis (Supplementary Fig. S7). We established that suppression of Pirh2 did not affect the HuR mRNA level. Noteworthy, in contrast to the expectation, attenuation of Pirh2 led to a moderate decrease of the HuR mRNA level, therefore strongly suggesting that the increased level of HuR observed in Pirh2 KD cells was due to the protein stabilization and not to the regulation of its transcription (Supplementary Fig. S7). 
It has been reported that c-Myc proto-oncogene sensitizes cells to heat-induced cell death ${ }^{40,41}$. Hence, downregulation of $\mathrm{c}-\mathrm{Myc}$ would be a plausible mechanism responsible for the survival of HeLa cells upon HS. In this respect, we have recently shown that Pirh2 was a positive regulator of c-Myc expression in $\mathrm{H} 1299$ cells, and that the attenuation of Pirh2 resulted in a decrease of c-Myc levels ${ }^{24}$. Notably, HuR is a negative regulator of c-Myc expression at the level of mRNA stability ${ }^{42}$. Taking these facts together, we hypothesized the existence of a putative regulatory loop, whereby Pirh2 stabilized c-Myc via the degradation of HuR, and the latter, in turn, inhibited the translation of c-Myc. To test this hypothesis, we ascertained whether Pirh2 KD simultaneously stabilized HuR and attenuated c-Myc, both in normal conditions and after HS. In agreement with our hypothesis, in Pirh2 KD cells we observed a modest, but reproducible, elevation of HuR levels concomitant with a decrease of the c-Myc level (Fig. 4E).

We also confirmed the involvement of c-Myc in the Pirh2-regulated heat-shock response. To this end, HeLa Pirh2 KD and scrambled control cells were transiently transfected with the c-Myc expression vector and the colony-formation abilities of these cell lines were compared before and after HS. In agreement with our hypothesis that the Pirh2/HuR interaction affects the HS response via $\mathrm{c}-\mathrm{Myc}$, we found that the forced expression of c-Myc sensitized HeLa cells to HS irrespective of the Pirh2 status (Supplementary Fig. S8).

\section{Pirh2 activates c-Myc expression via downregulation of HuR}

As $\mathrm{HuR}$ was shown to downregulate $\mathrm{c}-\mathrm{Myc}$ via recruiting the RNA miRNA-induced silencing complex (RISC) to its $\mathrm{mRNA}^{42}$, we first examined the effect of Pirh2 on c-Myc, on the level of mRNA in H1299 cells. When mRNA levels of c-Myc were compared between H1299 cells with constitutive Pirh2 OE and control cells (ctrl), it became apparent that the elevated c-Myc mRNA level correlated with overexpression of Pirh2 (Fig. 5A). On the contrary, the c-Myc mRNA level was attenuated in shRNA-mediated KD of Pirh2 (Pirh2 KD) (Fig. 5B). Interestingly, genotoxic stress caused by $1 \mu \mathrm{M}$ doxorubicin treatment for $16 \mathrm{~h}$ had no effect on the ability of Pirh2 to stabilize the level of c-Myc as judged by RT-PCR (compare Fig. 5A, B). The same pattern was observed on the protein level (Fig. $5 \mathrm{C}, \mathrm{D}$ ).

Considering that $\mathrm{c}-\mathrm{Myc}$ is a potent cell cycle regulator, we performed cell cycle analysis using isogenic cell lines that differed in the Pirh2 status. To induce genotoxic stress, cells were treated with Etoposide for the indicated time period. Pirh2-mediated G2/M block was observed in cells treated with $100 \mu \mathrm{M}$ etoposide for $24 \mathrm{~h}$ (Fig. 5E). On the contrary, Pirh2 KD cells demonstrated the opposite effect (Fig. 5F).

\section{Pirh2 prevents HuR binding to c-Myc mRNA}

As it was noted above, $\mathrm{HuR}$ was shown to downregulate c-Myc via recruiting the RISC complex to its $\mathrm{mRNA}^{42}$. To additionally confirm our hypothesis that Pirh2 augments c-Myc levels through downregulation of $\mathrm{HuR}$, we performed RIP assay. We used H1299 cell lines with attenuated expression of either Pirh2 or HuR. H1299 cells with scrambled shRNA were used as control (Fig. 6A). As HuR was reported to preferentially bind $3^{\prime}$-UTRs of its target genes, we used two pairs of primers for RT-PCR analysis specific for the coding region (CDS) and $3^{\prime}$-UTR of c-myc (Fig. 6B). We reasoned that the suppression of Pirh2 would increase the cellular level of HuR and hence augment the amount of c-Myc mRNA co-precipitated with $\mathrm{HuR}$. In fact, KD of Pirh2 promoted the binding of cMyc mRNA to HuR. Importantly, the most pronounced difference in binding was observed in the c-myc $3^{\prime}$-UTR region compared to c-myc CDS (Fig. 6C). These results correlate with the previously published data about the HuR-binding specificity ${ }^{42}$. We used HuR KD cells as control and demonstrated the decreased amounts of cMyc mRNA precipitation (Fig. 6C). Interestingly, in Pirh2 KD cells we also observed a moderate decrease of $\mathrm{HuR}$ mRNA. Thus, we confirmed that the effect of Pirh2 on cMyc expression is mediated via the Pirh2-dependent regulation of $\mathrm{HuR}$.

\section{Pirh2 stimulates proliferation of $\mathrm{H} 1299$ cell and cooperates with c-Myc in lung cancer progression}

To demonstrate the functional effect of Pirh2dependent attenuation of HuR, we tested the effect of Pirh2 expression on proliferation of H1299 non-small cell lung cancer cells.

To measure the proliferation rate of H1299 cells with different status of Pirh2, we performed real-time monitoring of cell growth using an xCELLigence system (Fig. $7 \mathrm{~A}, \mathrm{~B})$. This approach is based on the continuous measurement of the impedance difference of metal bottom of the cell chambers upon the cell growth and spreading. Therefore, this system provides the opportunity to monitor the number of cells attached to the bottom well in real time. We noticed that Pirh2 OE increased the proliferation rate of H1299 cells (compare H1299 Pirh2 OE vs. H1299 ctrl) (Fig. 7A). On the contrary, KD of Pirh2 retarded the proliferation rate of cells (H1299 Pirh2 KD vs. H1299 scrambled) (Fig. 7B).

As the proliferation activity is one of the major characteristics of cancer cells, we examined whether Pirh2 and c-Myc expression levels correlate with survival rates of lung cancer patients. Indeed, as shown in Fig. 7C, reduced expression of $M y c$ and $R C H Y 1$ genes, positively correlated with the survival of lung cancer patients. On the contrary, high level of HuR (Elavl1) expression within the same 


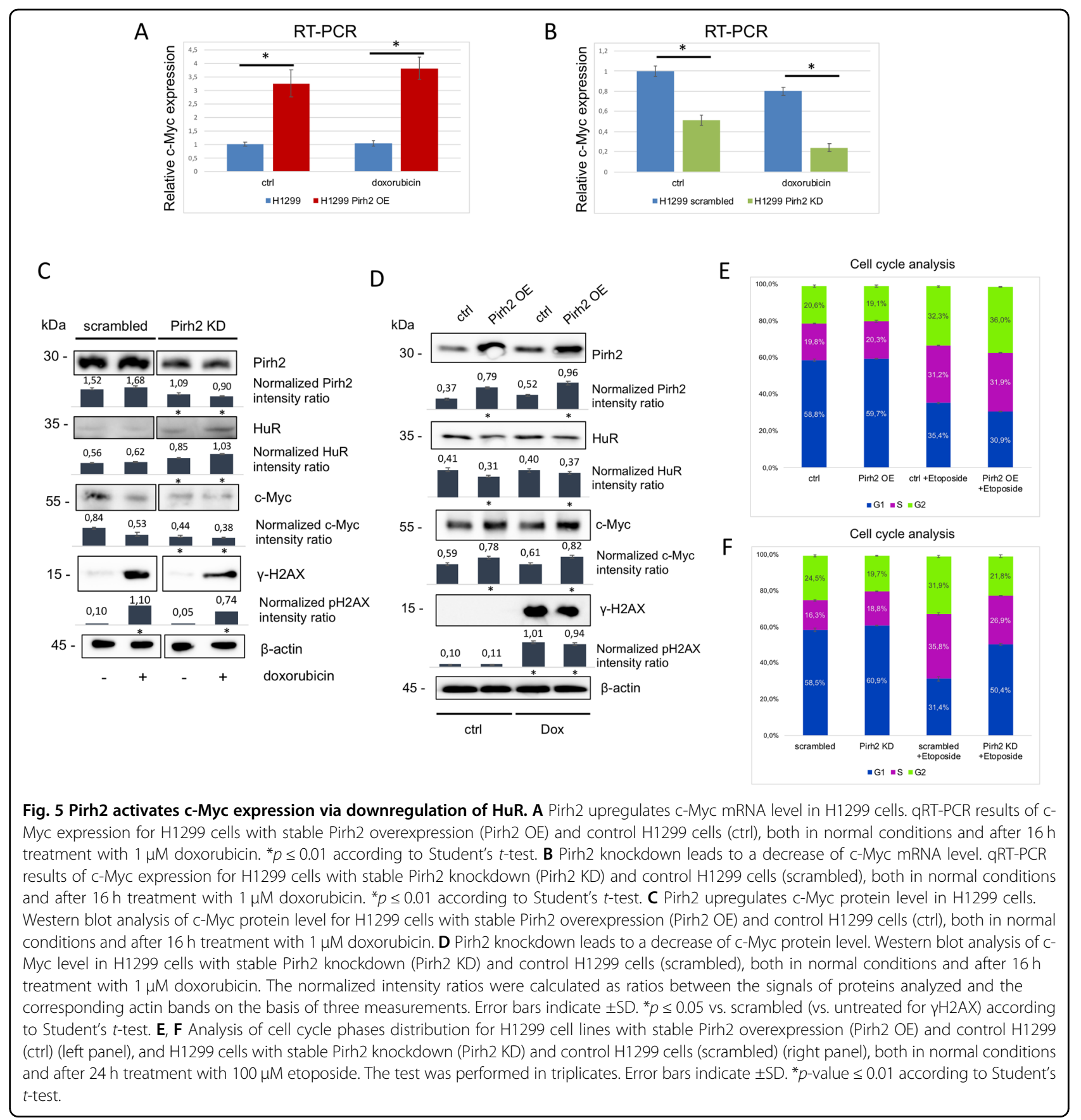

GEO dataset correlated with the prolonged survival of cancer patients (Fig. 7D).

Thus, we showed that increased levels of Pirh2 and cMyc displayed the oncogenic effects using both the cellular model of lung cancer and the bioinformatics approach using the patient-derived gene expression data.

\section{Discussion}

A wealth of data available in the literature strongly suggests that E3 ligase Pirh2 exerts its functions both in p53-dependent and independent ways ${ }^{43,44}$. Furthermore, Pirh2 can play opposite roles in tumorigenesis depending on the cellular context. For example, overexpression of Pirh2 inhibits epithelial to mesenchymal transition by ubiquitinating Twist and sending the latter for proteasome-dependent degradation ${ }^{45}$. Furthermore, Pirh2 is able to attenuate the nuclear factor- $\mathrm{kB}$ (NF- $\mathrm{kB}$ ) pathway in bortezomib-resistant multiple myeloma cells via ubiquitination of $\mathrm{pIKBa}$ and IKKa, the two critical regulators of NF- $\mathrm{KB}^{46}$. On the other hand, several studies 


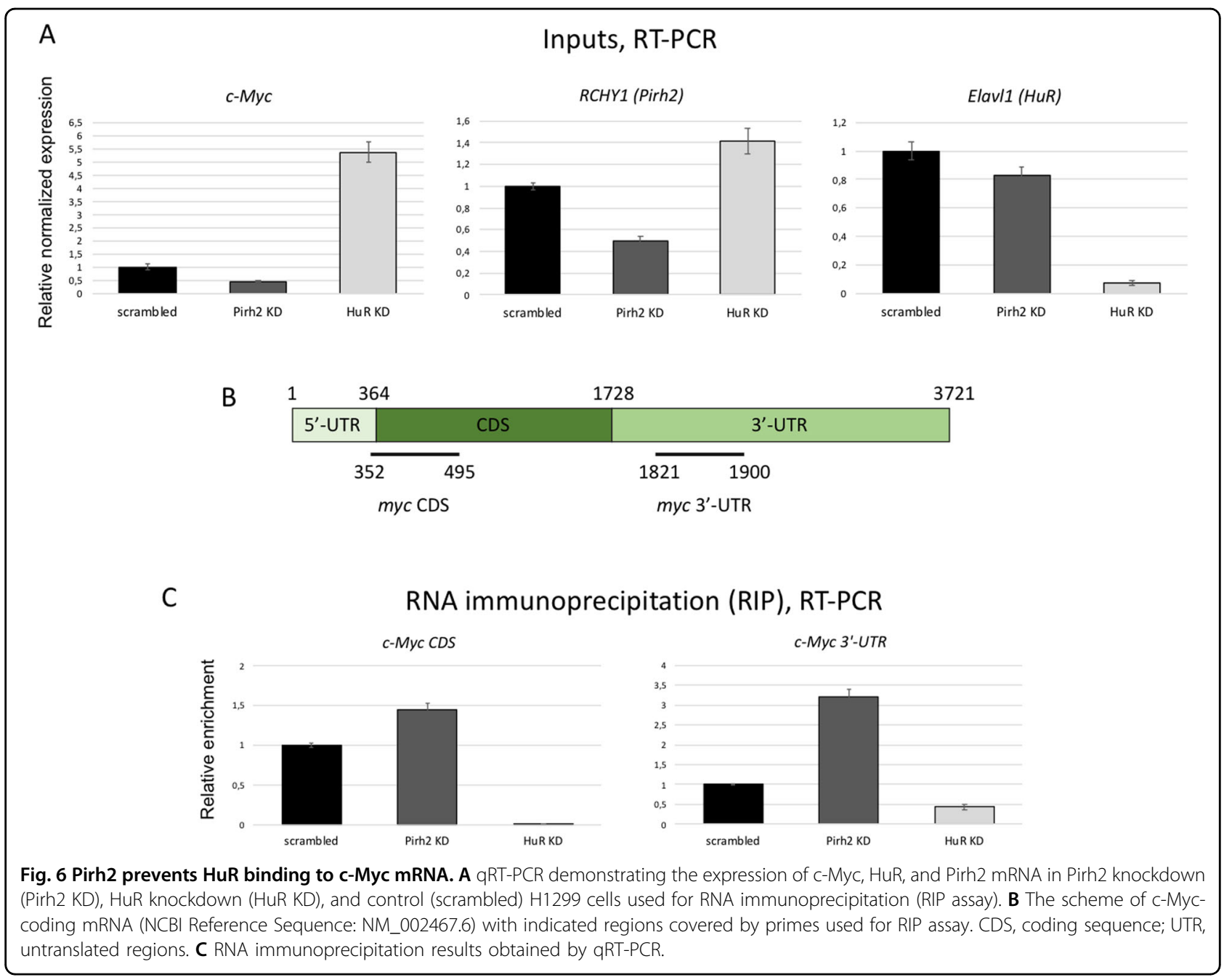

including our own work, have shown that Pirh2 promotes tumorigenesis by degrading p53, p63, p73, Chk2, p2 $7^{\text {kip1 }}$, and other important tumor suppressor proteins ${ }^{3,4,6,15,17}$.

Cellular functions of the protein of interest are often governed by its interacting partners ${ }^{33}$. Therefore, it seemed important to identify new interacting partners of Pirh2, to elucidate its physiological role in cells. E3 ligases, including the Pirh2 protein, often interact with their substrates only transiently. Therefore, we chose mild GST-pulldown conditions to capture as many transiently Pirh2-associated proteins as possible. Subsequently, the proteomic approach has identified 225 Pirh2-interacting proteins. Importantly, a large portion of Pirh2 interactors belonged to RNA processing/splicing factors (Fig. 1). Among those, we focused on the RNA-binding protein, HuR (ELAVL1), because the latter has previously been reported to participate in the p53 regulation.

The HuR protein is a product of the ELAVL1 gene and one of the members of the embryonic lethal abnormal vision (ELAV)/Hu family. In most cases, $\mathrm{HuR}$ binds to Uand AU-rich motifs in the $3^{\prime}$-UTR region of mRNAs and differentially affects their stability ${ }^{47,48}$. By regulating the mRNA stability, HuR plays various roles in the cell including its participation in mRNA nuclear cytoplasmic shuttling ${ }^{49}$ and splicing ${ }^{50,51}$. In the cytoplasm, HuR regulates the stability of target mRNAs, both positively and negatively. For example, HuR increases the half-life of several mRNAs, including MMP9, cyclins A, B1, D1, and GSK3 $\beta^{52-54}$. On the contrary, other mRNAs (e.g., p27, RhoB, and c-Myc) are either repressed or degraded by $\mathrm{HuR}^{42,55,56}$. Specifically, HuR inhibits the expression of CDKN1B by binding the $5^{\prime}$-UTR to the gene in the region of internal ribosomal entry site (IRES), thereby attenuating the process of CDKN1B translation ${ }^{56}$. On the other hand, HuR mediates degradation of c-Myc mRNA by recruiting RISC loaded with microRNA let-7, which in turn targets the c-Myc $3^{\prime}-\mathrm{UTR}^{42}$. In this respect, it is important to note that we have previously shown Pirh2mediated upregulation of c-Myc expression in non-small 
A

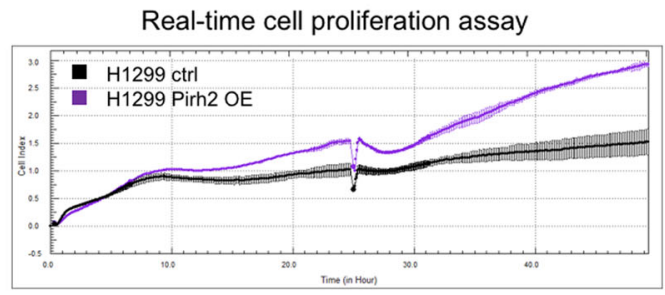

C

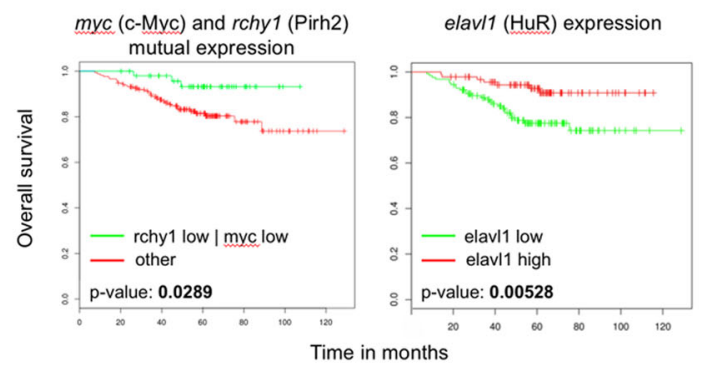

GEO dataset ID: GSE31210

GEO dataset Type: Lung cancer

GEO dataset Title: Gene expression data for pathological stage I-II lung adenocarcinomas
B

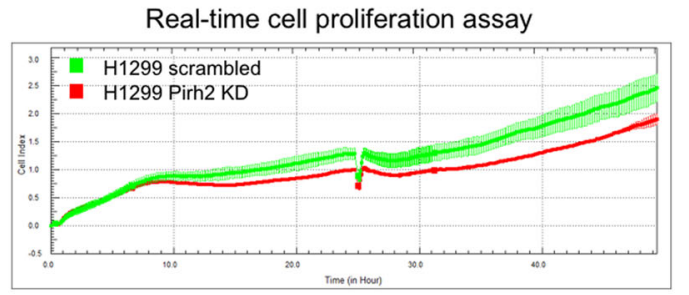

D

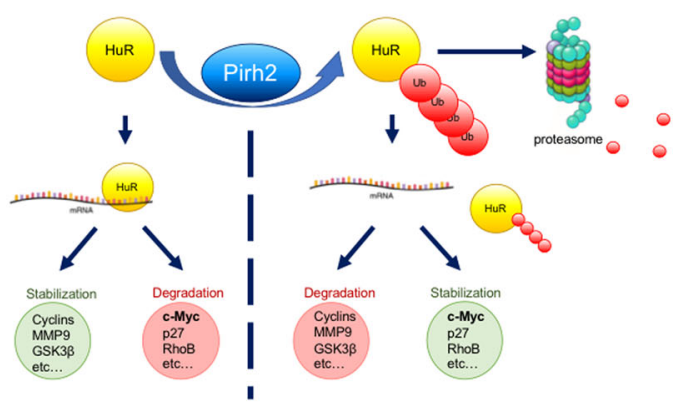

Fig. 7 Pirh2 activates H1299 cell proliferation and cooperates with c-Myc in lung cancer progression. A Pirh2 stimulates proliferation of H1299 cells. Cell index graphs demonstrating the proliferation registered by xCelligence system for H1299 cells with stable Pirh2 overexpression (Pirh2 OE) and control H1299 cells (ctrl). B Pirh2 knockdown suppresses proliferation of H1299 cells. Cell index graphs registered by xCelligence system for H1299 cells with stable Pirh2 knockdown (Pirh2 KD) and control H1299 cells (scrambled). C The bioinformatics analysis of the effect of simultaneous rchyl and myc genes (coding Pirh2 and c-Myc, respectively), and elavl1 gene (coding HuR protein) expression on lung adenocarcinoma patients' (GEO dataset ID: GSE31210) survival probability. P-values are indicated. D Graphical scheme summarizing the role of Pirh2 in HuR protein stability regulation. Pirh2 ubiquitinates HuR that leads to HuR proteasomal degradation and influence on HuR target mRNA stability.

lung carcinoma cells, both on the level of its mRNA and protein translation $^{24}$.

In line with this observation, in the present study we showed that Pirh2 regulated c-Myc via affecting the stability of HuR by ubiquitinating the latter. In contrast, ablation of Pirh2 caused stabilization of $\mathrm{HuR}$, which in turn decreased the level of c-Myc expression in H1299 cells.

To date, only one E3 ligase,TRIM21, has been reported to ubiquitinate $\mathrm{HuR}^{57}$. This TRIM21-dependent ubiquitination caused HuR degradation as part of the UV response and subsequently affected p53 accumulation under UV-induced stress. In addition to that study, we demonstrated the universal Pirh2-dependent mechanism of $\mathrm{HuR}$ attenuation, both under normal condition and under cellular stress caused by heat shock or DNA damage.

Taking into account the number of HuR mRNA targets playing central roles in key cellular processes, it is not surprising that HuR expression is shown to be a biomarker for numerous cancers ${ }^{58-60}$. In particular, elevated cytoplasmic HuR presence was shown to be a marker of poor prognosis and cancer aggressiveness for bladder cancer, meningioma, lung cancer, and esophageal squamous cell carcinoma ${ }^{61-64}$. However, high expression levels of HuR may also have a favorable prognostic value, e.g., for breast cancer ${ }^{65}$, which may be associated with HuR-mediated stabilization of p53 and hence activation of its proapoptotic downstream targets ${ }^{6-68}$.

Using the bioinformatics approach, we revealed that low Pirh2 and c-Myc levels and high HuR level correlated with better survival prognosis of patients with lung adenocarcinoma (Fig. 7C). To corroborate our results, we propose a model (Fig. 7E), whereby Pirh2 attenuates the protein stability of HuR and hence modulates levels of expression of HuR target genes. Future studies should reveal the effect of Pirh2 on other mRNA targets of HuR.

\section{Acknowledgements}

We thank Drs M. Gorospe (National Institutes of Health, Baltimore, USA) and S. Benchimol (York University, Toronto, Ontario, Canada) for kindly providing HuR- and Pirh2-expressing plasmids, respectively. O.F., A.P., and E.T. acknowledge the support from RSF grant number 18-75-10076. A.D., O. Shuvalov, and N.B. acknowledge the support from RFBR grant number 18-2909144. A.D., O. Shuvalov, O.F., O. Semenov, and N.B. appreciate the support of the grant from the Russian Government Program for the Recruitment of the leading scientists into the Russian Institutions of Higher Education \#14. W03.31.0029. 


\section{Author details}

${ }^{1}$ Institute of Cytology, Russian Academy of Sciences, 194064 St Petersburg, Russian Federation. ${ }^{2}$ Almazov National Medical Research Centre, Institute of Hematology, 197341 St Petersburg, Russian Federation. ${ }^{3}$ School of Life Sciences, University of Warwick, Coventry CV4 7AL, UK. ${ }^{4}$ Moscow Institute of Physics and Technology, 141700 Dolgoprudny, Moscow Region, Russian Federation

\section{Author contributions}

A.D., A.P., O.F., and O. Shuvalov designed and performed the experiments, and prepared figures and table. A.K. and E.V. performed the bioinformatic analysis. A.B. performed the mass spectrometry analysis. E.T. participated in c-Myc overexpression experiments. O. Semenov participated in MS data processing. A.D. wrote the manuscript. N.B. executed general leadership, pointed out directions of the research, and performed editing of the manuscript.

\section{Ethics statement}

Non-applicable.

\section{Conflict of interest}

The authors declare no competing interests.

\section{Publisher's note}

Springer Nature remains neutral with regard to jurisdictional claims in published maps and institutional affiliations.

Supplementary information The online version contains supplementary material available at https://doi.org/10.1038/s41419-021-03871-w.

Received: 5 January 2021 Revised: 18 May 2021 Accepted: 24 May 2021 Published online: 05 June 2021

\section{References}

1. Beitel, L. et al. Cloning and characterization of an androgen receptor $\mathrm{N}$ terminal-interacting protein with ubiquitin-protein ligase activity. J. Mol Endocrinol. 29, 41-60 (2002).

2. Sheng, Y. et al. Molecular basis of Pirh2-mediated p53 ubiquitylation. Nat Struct. Mol. Biol. 15, 1334 (2008).

3. Leng, R. P. et al. Pirh2, a p53-induced ubiquitin-protein ligase, promotes p53 degradation. Cell 112, 779-791 (2003).

4. Jung, Y.-S., Qian, Y. \& Chen, X. The p73 tumor suppressor is targeted by Pirh2 RING finger E3 ubiquitin ligase for the proteasome-dependent degradation. J. Biol. Chem. 286, 35388-35395 (2011).

5. Wu, H., Abou, Z. R., Flores, E. R. \& Leng, R. P. Pirh2, a ubiquitin E3 ligase, inhibits p73 transcriptional activity by promoting its ubiquitination. Mol. Cancer Res. 9 , 1780-1790 (2011)

6. Jung, Y.-S., Qian, Y., Yan, W. \& Chen, X. Pirh2 E3 ubiquitin ligase modulates keratinocyte differentiation through p63. J. Invest. Dermatol. 133, 1178-p87 (2013).

7. Tai, E. Characterization of the E3 Ubiquitin Ligase Pirh2, http://hdl.handle.net/ 1807/24889 (2010).

8. Morgunkova, A. \& Barlev, N. A. Lysine methylation goes global. Cell Cycle $\mathbf{5}$, 1308-1312 (2006)

9. Yan, W. et al. Arsenic suppresses cell survival via Pirh2-mediated proteasomal degradation of $\triangle$ Np63 protein. J. Biol. Chem. 288, 2907-2913 (2013).

10. Yan, W., Jung, Y.-S., Zhang, Y., Chen, X. Arsenic trioxide reactivates proteasomedependent degradation of mutant p53 protein in cancer cells in part via enhanced expression of Pirh2 E3 ligase. PLOS ONE 9, e103497 (2014).

11. Daks, A. et al. Tumor suppressor p63 regulates Pirh2 ubiquitin ligase expression. Cell Tissue Biol. 10, 202-205 (2016).

12. Logan, I. R. et al. Human $\mathrm{PIRH} 2$ enhances androgen receptor signaling through inhibition of histone deacetylase 1 and is overexpressed in prostate cancer. Mol. Cell. Biol. 26, 6502-6510 (2006).

13. Hattori, T. et al. Pirh2 promotes ubiquitin-dependent degradation of the cyclin-dependent kinase inhibitor p27Kip1. Cancer Res. 67, 10789-10795 (2007).
14. Jung, Y.-S., Liu, G. \& Chen, X. Pirh2 E3 ubiquitin ligase targets DNA polymerase eta for $20 \mathrm{~S}$ proteasomal degradation. Mol. Cell. Biol. 30, 1041-1048 (2010).

15. Bohgaki, $\mathrm{M}$. et al. The E3 ligase $\mathrm{PIRH} 2$ polyubiquitylates $\mathrm{CHK} 2$ and regulates its turnover. Cell Death Differ. 20, 812-822 (2013).

16. Duan, W. et al. Expression of Pirh2, a newly identified ubiquitin protein ligase, in lung cancer. J. Natl Cancer Inst. 96, 1718-1721 (2004).

17. Shimada, M. et al. High expression of Pirh2, an E3 ligase for p27, is associated with low expression of p27 and poor prognosis in head and neck cancers. Cancer Sci. 100, 866-872 (2009).

18. Bao, Y., Wu, X., Yuan, D., Shi, W. \& Shi, J. High expression of Pirh2 is associated with poor prognosis in glioma. Cell. Mol. Neurobiol. 37, 1501-1509 (2017).

19. Wang, X. M., Yang, L. Y., Guo, L., Fan, C. \& Wu, F. p53-induced RING-H2 protein a novel marker for poor survival in hepatocellular carcinoma after hepatic resection. Cancer 115, 4554-4563 (2009).

20. Zheng, J. et al. Inverse correlation between Naa10p and Pirh2 expression and the combined prognostic value in oral squamous cell carcinoma patients. J. Oral. Pathol. Med. 48, 686-695 (2019).

21. Hakem, A. et al. Role of Pirh2 in mediating the regulation of p53 and c-Myc PLoS Genet. 7, e1002360 (2011).

22. Lezina, L. et al. KMTase Set7/9 is a critical regulator of E2F1 activity upon genotoxic stress. Cell Death Differ. 21, 1889-1899 (2014).

23. Moffat, J. et al. A lentiviral RNAi library for human and mouse genes applied to an arrayed viral high-content screen. Cell 124, 1283-1298 (2006).

24. Daks, A. et al. E3 ubiquitin ligase Pirh2 enhances tumorigenic properties of human non-small cell lung carcinoma cells. Genes Cancer 7, 383 (2016).

25. Weber, K., Bartsch, U., Stocking, C. \& Fehse, B. A multicolor panel of novel lentiviral "gene ontology"(LeGO) vectors for functional gene analysis. Mol. Ther. 16, 698-706 (2008)

26. Fedorova, O. A. et al. Proteomic analysis of the 205 proteasome (PSMA3)interacting proteins reveals a functional link between the proteasome and mRNA metabolism. Biochem. Biophys. Res. Commun. 416, 258-265 (2011).

27. Tsimokha, A. S., Zaykova, J. J., Bottrill, A. \& Barlev, N. A. Extracellular proteasomes are deficient in 195 subunits as revealed by iTRAQ quantitative proteomics. J. Cell. Physiol. 232, 842-851 (2017).

28. Vasileva, E. et al. KMT Set7/9 is a new regulator of Sam68 STAR-protein. Biochem. Biophys. Res. Commun. 525, 1018-1024 (2020).

29. Fedorova, O. et al. Orphan receptor NR4A3 is a novel target of p53 that contributes to apoptosis. Oncogene 38, 2108-2122 (2019).

30. Rada, M. et al. BTK blocks the inhibitory effects of MDM2 on p53 activity. Oncotarget 8, 106639 (2017).

31. Shuvalov, O. et al. Nutlin sensitizes lung carcinoma cells to interferon-alpha treatment in MDM2-dependent but p53-independent manner. Biochem. Biophys. Res. Commun. 495, 1233-1239 (2018).

32. Amelio, I. et al. SynTarget: an online tool to test the synergetic effect of genes on survival outcome in cancer. Cell Death Differ. 23, 912 (2016).

33. Antonov, A. et al. PPISURV: a novel bioinformatics tool for uncovering the hidden role of specific genes in cancer survival outcome. Oncogene 33, 1621 (2014).

34. Gallouzi, I.-E. et al. HuR binding to cytoplasmic mRNA is perturbed by heat shock. Proc. Natl Acad. Sci. USA 97, 3073-3078 (2000).

35. GALLOUZI, I.-E., BRENNAN, C. M. \& STEITZ, J. A. Protein ligands mediate the CRM1-dependent export of HuR in response to heat shock. RNA 7, 1348-1361 (2001).

36. Amadio, M. et al. Post-transcriptional regulation of HSP70 expression following oxidative stress in SH-SY5Y cells: the potential involvement of the RNAbinding protein HuR. Curr. Pharm. Des. 14, 2651-2658 (2008).

37. Abdelmohsen, $\mathrm{K}$. et al. Ubiquitin-mediated proteolysis of HuR by heat shock. EMBO J. 28, 1271-1282 (2009).

38. Kühl, N. \& Rensing, L. Heat shock effects on cell cycle progression. Cell. Mol. Life Sci. 57, 450-463 (2000).

39. Sottile, M. L. \& Nadin, S. B. Heat shock proteins and DNA repair mechanisms: an updated overview. Cell Stress Chaperones. 23, 303-315 (2018).

40. Li, W. X., Chen, C., Ling, C. \& Li, G. C. Apoptosis in heat-induced cell killing: the protective role of hsp-70 and the sensitization effect of the c-myc gene. Radiat. Res. 145, 324-330 (1996).

41. Prendergast, G. C. Mechanisms of apoptosis by c-Myc. Oncogene 18, 2967-2987 (1999).

42. Kim, H. H. et al. HuR recruits let-7/RISC to repress c-Myc expression. Genes Dev. 23, 1743-1748 (2009) 
43. Halaby, M.j, Hakem, R. \& Hakem, A. Pirh2: an E3 ligase with central roles in the regulation of cell cycle, DNA damage response, and differentiation. Cell Cycle 12, 2733-2737 (2013).

44. Jung, Y.-S., Qian, Y. \& Chen, X. Pirh2 RING-finger E3 ubiquitin ligase: its role in tumorigenesis and cancer therapy. FEBS Lett. 586, 1397-1402 (2012).

45. Yang-Hartwich, Y. et al. p53-Pirh2 complex promotes Twist1 degradation and inhibits EMT. Mol. Cancer Res. 17, 153-164 (2019).

46. Yang, L. et al. Pirh2 mediates the sensitivity of myeloma cells to bortezomib via canonical NF-kB signaling pathway. Protein Cell. 9, 770-784 (2018).

47. de Silanes, I. L., Zhan, M., Lal, A., Yang, X. \& Gorospe, M. Identification of a target RNA motif for RNA-binding protein HuR. Proc. Natl Acad. Sci. USA 101 2987-2992 (2004).

48. Uren, P. J. et al. Genomic analyses of the RNA-binding protein Hu antigen R (HuR) identify a complex network of target genes and novel characteristics of its binding sites. J. Biol. Chem. 286, 37063-37066 (2011).

49. Fan, X. C. \& Steitz, J. A. HNS, a nuclear-cytoplasmic shuttling sequence in HuR. Proc. Natl Acad. Sci. USA 95, 15293-15298 (1998).

50. Akaike, Y. et al. HuR regulates alternative splicing of the TRA2 $\beta$ gene in human colon cancer cells under oxidative stress. Mol. Cell. Biol. 34, 2857-2873 (2014)

51. Izquierdo, J. M. Hu antigen R (HuR) functions as an alternative pre-mRNA splicing regulator of Fas apoptosis-promoting receptor on exon definition. J. Biol. Chem. 283, 19077-19084 (2008).

52. Hoffman, O. et al. Detrimental ELAVL-1/HuR-dependent GSK3 $\beta$ mRNA stabilization impairs resolution in acute respiratory distress syndrome. PLOS ONE $\mathbf{1 2}$ e0172116 (2017).

53. Wang, W., Caldwell, M. C., Lin, S., Furneaux, H. \& Gorospe, M. HuR regulates cyclin $A$ and cyclin B1 mRNA stability during cell proliferation. EMBO J. 19, 2340-2350 (2000).

54. Zybura-Broda, K. et al. HuR (Elavl1) and HuB (Elavl2) stabilize matrix metalloproteinase-9 mRNA during seizure-induced Mmp-9 expression in neurons. Front. Neurosci. 12, 224 (2018)

55. Glorian, V. et al. HuR-dependent loading of miRNA RISC to the mRNA encoding the Ras-related small GTPase RhoB controls its translation during UV-induced apoptosis. Cell Death Differ. 18, 1692-1701 (2011).

56. Kullmann, M., Göpfert, U., Siewe, B. \& Hengst, L. ELAV/Hu proteins inhibit p27 translation via an IRES element in the p27 5' UTR. Genes Dev. 16, 3087-3099 (2002).
57. Guha, A. et al. Integrated regulation of HuR by translation repression and protein degradation determines pulsatile expression of p53 under DNA damage. iscience 15, 342-359 (2019).

58. Abdelmohsen, K. \& Gorospe, M. Posttranscriptional regulation of cancer traits by HuR. Wiley Interdiscip. Rev. RNA 1, 214-229 (2010).

59. Schultz, C. W., Preet, R., Dhir, T., Dixon, D. A. \& Brody, J. R. Understanding and targeting the disease-related RNA binding protein human antigen R (HuR). Wiley Interdiscip. Rev. RNA 11, e1581 (2020).

60. Wang, J. et al. Multiple functions of the RNA-binding protein HuR in cancer progression, treatment responses and prognosis. Int. J. Mol. Sci. 14, 10015-10041 (2013).

61. Gauchotte, G. et al. Cytoplasmic overexpression of RNA-binding protein HuR is a marker of poor prognosis in meningioma, and HuR knockdown decreases meningioma cell growth and resistance to hypoxia. J. Pathol. 242, 421-434 (2017).

62. Miyata, Y. et al. High expression of HuR in cytoplasm, but not nuclei, is associated with malignant aggressiveness and prognosis in bladder cancer. PLOS ONE 8, e59095 (2013).

63. Wang, J., Wang, B., Bi, J. \& Zhang, C. Cytoplasmic HuR expression correlates with angiogenesis, lymphangiogenesis, and poor outcome in lung cancer. Med. Oncol. 28, 577-585 (2011).

64. Zhang, $\mathrm{C}$. et al. Cytoplasmic expression of the ELAV-like protein HuR as a potential prognostic marker in esophageal squamous cell carcinoma. Tumor Biol. 35, 73-80 (2014)

65. Yuan, Z., Sanders, A. J., Ye, L., Wang, Y. \& Jiang, W. G. Prognostic value of the human antigen $\mathrm{R}$ (HuR) in human breast cancer: high level predicts a favourable prognosis. Anticancer Res. 31, 303-310 (2011).

66. Nakamura, H. et al. Cooperative role of the RNA-binding proteins $\mathrm{Hzf}$ and HuR in p53 activation. Mol. Cell. Biol. 31, 1997-2009 (2011).

67. Zou, T. et al. Polyamine depletion increases cytoplasmic levels of RNA-binding protein HuR leading to stabilization of nucleophosmin and p53 mRNAs. J. Biol. Chem. 281, 19387-19394 (2006).

68. Mazan-Mamczarz, K. et al. RNA-binding protein HuR enhances p53 translation in response to ultraviolet light irradiation. Proc. Natl Acad. Sci. USA 100 8354-8359 (2003). 